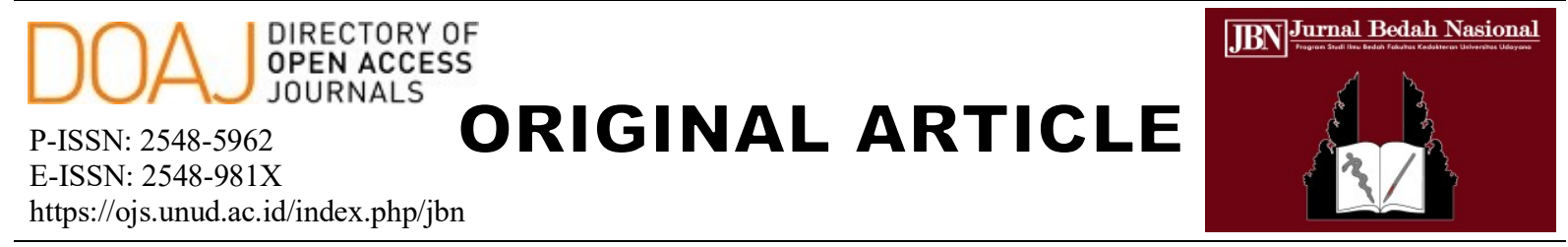

\title{
Validity of Barium Enema as Hirschsprung's Disease Diagnostic Tools for Infant in Sanglah Hospital Denpasar
}

\author{
I Made Darmajaya*, I Ketut Subhawa
}

Department of Pediatric Surgery, Universitas Udayana Medical School/Sanglah Hospital Denpasar Bali.

*Corresponding author: drdarmajaya@gmail.com.

\begin{abstract}
Aim: To determine the validity (sensitivity, specificity, likelihood ratio) of barium enema as Hirschsprung's disease diagnostic tool in infants. Methods: This study was a diagnostic test of barium enema compared with postoperative histopathology examinations as the gold standard. The population were all patients with indigestion symptom be discovered at the pediatric surgical clinic of Sanglah Hospital Denpasar. The sampling method was consecutive sampling, satisfy inclusion criteria $(\leq 12$ months old, indicate classic symptoms of Hirschsprung's disease). The total sample of the study was 52 patients. Sensitivity, specificity, positive and negative predictive value were analysed using crosstabulation test of barium enema and postoperative histopathology. Results: A total of 52 patients were evaluated during the study period, mean of age was 3.31 months old, and boys:girls (75\%:25\%). Based on symptoms, $98.08 \%$ of patients were delayed release of meconium more than $24-48$ hours and abdominal distention. Among all the patients reviewed, sensitivity, specificity, positive and negative predictive value of barium enema for diagnostic of Hirschsprung's disease was 95.5\%, 87.5\%, 97.7\%, and $77.8 \%$. Conclusion: Barium enema can be used as an early diagnostic tool for infants suspected of Hirschsprung's disease.
\end{abstract}

Keywords: barium enema, diagnostic test, infant, Hirschsprung's disease.

DOI: https://doi.org/10.24843/JBN.2021.v05.i01.p03

\section{INTRODUCTION}

Hirschsprung's disease is a congenital intestinal motility disease characterized by the absence of parasympathetic ganglion cells in the myenteric and sub-mucosal plexus of the distal intestine. The etiology of this disease is thought to be caused by multifactorial causes. Failure to migrate of the neural crest cells to the craniocaudal part of the distal colon is considered to be the main embryological abnormality that causes Hirschsprung's disease. In addition, genetic disorders in the form of mutations in the Ret gene and endothelin $\mathrm{B}$ receptor gene are also associated as the cause of this disease. ${ }^{1}$

The incidence of this disease is 1: 5000 live births. $^{2}$ Epidemiologically, Hirschsprung's disease is found to be four times more common in males than in females. ${ }^{3}$ Several studies stated that a higher risk (12.4\% -33\%) occurs in patients who have sibling with total colonic involvement. Approximately $25 \%$ of newborn intestinal obstruction is caused by Hirschsprung's disease. ${ }^{4}$ Based on the location of the disorder, nearly $90 \%$ of the transition points are in the rectosigmoid colon (shortsegment aganglionosis). ${ }^{5}$

Down syndrome (trisomy 21) is the chromosomal disorder most associated with Hirschsprung's disease (about 10\% of all sufferers). Some other conditions that are suspected of being related to this disease include hydrocephalus, bladder diverticulum, Meckels diverticulum, anal imperforation, ventricular septal defect, renal agenesis, 
cryptorchidism, Waardenburg's syndrome, neuroblastoma, and Ondine's curse. ${ }^{6}$

Based on the clinical picture, about $90 \%$ of patients in the first month of life show symptoms of no meconium discharge in the first 24 hours of life, bowel movement disorders, refuse to feed, and progressive abdominal distension. Digital rectal examination can be done to determine the strength of the anal sphincter and explore the fecal and gas. ${ }^{7}$ Symptoms of the disease that are not definitive enough and the need for objective evidence to establish a diagnosis, causes Hirschsprung's disease to be one of the most difficult pediatric surgical diseases to diagnose. ${ }^{4,8}$

Early diagnosis will determine the morbidity and mortality rates of a disease. In Hirschsprung's disease, complications that can occur include constipation, fecal impaction which continues in a life-threatening condition, namely Hirschsprung-associated enterocolitis (HAEC). The HAEC morbidity rate is $15 \%-50 \%$ and the mortality rate reaches $20 \%-50 \%{ }^{9}$ The earlier the diagnosis is established it affects the selection of definitive surgery to be 1 stage compared to 3 stages if the patient comes late. This will provide an advantage over patients diagnosed and operated on at an older age with multistage surgery will have more frequent digestive problems. Another advantage is that it reduces the cost of care because the length of stay in the hospital will be shorter, and also provides better psychosocial benefits. ${ }^{1}$

The gold standard examination for diagnosis of Hirschsprung's disease is a fullthickness rectal biopsy (sensitivities 100\%). However, not all hospitals, especially regional hospitals, have facilities to carry out these examinations. In addition, this procedure is invasive and complicates the procedure in the form of perforation, bleeding, and infection. Therefore, in daily clinical practice there are examinations that can be used as a diagnosis tool for Hirschsprung's disease, namely barium enema and anorectal manometry. ${ }^{10-12}$

From the two types of investigations above, barium enema is more widely used than anorectal manometry. This is because barium enema is easier to perform, is minimally invasive, can be done in almost all regional hospitals, and has higher sensitivity and specificity. Barium enema has a sensitivity of $70-75 \%$ (reaching almost $100 \%$ using 24 -hour delayed abdominal radiographs) and a specificity of $70-80 \%$ whereas anorectal manometry has a sensitivity of $60 \%-70 \%$ and a specificity of $65 \%-70 \%$ across all age groups. ${ }^{13-15}$

The purpose of this study was to determine the validity of barium enema as a diagnostic tool for Hirschsprung's disease in infants. The study can be used as a reference for clinicians in the selection of initial diagnostic measures in patients with the infant age group with clinics that lead to Hirschsprung's disease.

\section{METHODS}

This study was an observational study with a diagnostic test to assess the validity of the diagnostic value of barium enema in detecting the presence of a lower gastrointestinal tract aganglionic segment, using a cross-sectional design. The sample size in this study was 52 samples of patients who met the inclusion and exclusion criteria from January to March 2016. Each patient sample was subjected to a barium enema examination and then a postoperative histopathological examination as the gold standard examination. Statistical analysis to compare validity between scores as a whole used the ROC analysis and significance was statistically assessed using the $p$ value at the 0.05 level of significance. The entire data analysed using statistical software Stata SE 12.1. 


\section{RESULTS}

From a total of 52 patients, the mean age was 3.31 months with an age range of 1 to 11 months (Table 1).

Table 1. Subjects' characteristic and research variables

\begin{tabular}{ll}
\hline Variable & $\mathbf{n}=\mathbf{5 2}$ \\
\hline Age, mean \pm SD & $3.31 \pm 2.98$ \\
min, max & $1-11($ age $)$ \\
Gender & \\
Male & $39(75 \%)$ \\
Female & $13(25 \%)$ \\
Symptoms & $39(75 \%)$ \\
Delayed of meconium & $51(98.08 \%)$ \\
Abdominal distention & $39(75 \%)$ \\
Bile vomit & \\
Barium Enema & $43(82.69 \%)$ \\
Positive & $9(17.31 \%)$ \\
Negative & $9(17.31 \%)$ \\
Barium Enema interpretation & \\
Not Hirschsprung's & \\
$\quad$ Disease & $40(76.92 \%)$ \\
Hirschsprung's Short & \\
$\quad$ Segment & $3(5.77 \%)$ \\
Hirschsprung's Long & \\
$\quad$ Segment & \\
Histopathology & $44(84.62 \%)$ \\
Positive & $8(15.38 \%)$ \\
Negative & \\
Surgery procedure & $2(3.85 \%)$ \\
Biopsy & $10(19.23 \%)$ \\
Duhamel & $24(46.15 \%)$ \\
Transanal Endorectal & \\
Pullthrough & $16(30.77 \%)$ \\
LATEP & \\
\hline
\end{tabular}

The distribution based on gender was 39 (75\%) for male and 13 (25\%) for female. Based on the patient's symptoms, it was found that $51(98.08 \%)$ patients experienced delays in expulsion of the meconium more than 2448 hours after birth, and 1 (1.92\%) patient had no delay in expelling the meconium. Symptoms of abdominal distension were also reported in $51(98.08 \%)$ patients while 1 (1.92\%) patient without abdominal distension. Symptoms of green vomiting (bile-stained vomiting) occurred in 39 (75\%) samples, and
$13(25 \%)$ samples without green vomiting symptoms.

Based on the results of the barium enema examination, $43(82.69 \%)$ patients were positive and $9(17.31 \%)$ patients were negative. From the barium enema examination, there were $40(76.92 \%)$ patients with the short segment type and 3 (5.77\%) patients with the long segment type, whereas the results of postoperative histopathological examination found $44(84.62 \%)$ patients with positive results of aganglion colon segment and $8(15.38 \%)$ patients with negative histopathology results.

Operative management in the sample including $2(3.85 \%)$ patients performed rectal biopsy, Duhamel procedure in $10(19.23 \%)$ patients, transanal endorectal pull-through surgery for $24(46.15 \%)$ patients, and laparoscopic assisted transanal endorectal pullthrough (LATEP) procedure in 16 (30.77\%) patients.

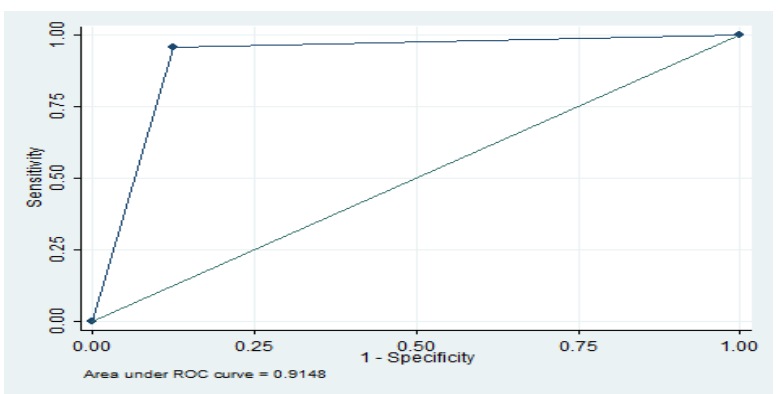

Figure 1. ROC graphic of barium enema to detect aganglion segment of lower gastrointestinal tract with histopathology examination as gold standard

Table 2. Validity test of barium enema to detect aganglion segment in lower gastrointestinal tract with histopathology examination as the gold standard

\begin{tabular}{|c|c|c|c|c|}
\hline & & \multicolumn{2}{|c|}{ Hirschsprung's } & \multirow[t]{2}{*}{ Total } \\
\hline & & + & - & \\
\hline Barium enema & + & 42 & 1 & 43 \\
\hline & - & 2 & 7 & 9 \\
\hline Total & & 44 & 8 & 52 \\
\hline
\end{tabular}


Based on the results of the analysis using the ROC curve (Figure 1), it shows that the ability of the barium enema examination in detecting the lower gastrointestinal tract aganglion segment is very good, this is indicated by the value of the area under ROC curve of more than 0.7 , which is 0.9148 .

Among all the patients reviewed, sensitivity, specificity, positive and negative predictive value of barium enema for diagnostic of Hirschsprung's disease was $95.5 \%, 87.5 \%, 97.7 \%$, and $77.8 \%$ (Table 2).

\section{DISCUSSION}

Based on the results of the analysis using the ROC curve, it shows that the ability of the barium enema examination in detecting Hirschsprung's disease in infants is very good, this is indicated by the value of the area under ROC curve of more than 0.7 , which is 0.9148 . The value above shows that the barium enema examination provides the true conclusion in determining Hirschsprung's disease by $91 \%$.

From the barium enema diagnostic test, a sensitivity of $95.5 \%$ was obtained, which means that $95.5 \%$ of infants suffering from Hirschsprung's disease can be detected by barium enema examination. This result is higher when compared to other barium enema studies. Overall, from previous studies, the sensitivity obtained for this barium enema examination was in the range of $70-75 \%$ (reaching almost 100\% using 24-hour delayed abdominal radiographs). Research by Abbas et al. ${ }^{11}$ found the sensitivity of barium enema in diagnosing Hirschsprung's disease by $75 \%$. While the results were not much different from a similar study conducted by Wong et al. ${ }^{15}$ which obtained a sensitivity for barium enema of $75 \%$, but using 24 -hours delayed abdominal radiographs, the sensitivity increased to $100 \%$. Similar results were also obtained in the study of Huang et al. ${ }^{5}$, which received a sensitivity of $95.65 \%$. The similarity of these results is probably due to the not much different age range of the samples and the similarity in the method of barium enema examination using 24-hour delayed abdominal radiographs, which in Sanglah General Hospital Denpasar are referred to as barium retention photos.

The specificity of the barium enema in this study was found to be $87.5 \%$, indicating that the barium enema examination was able to determine $87.5 \%$ of subjects who did not have Hirschsprung's Disease among the infant group suspected of having Hirschsprung's Disease. This result is higher than previous studies. Research by Huang et al. ${ }^{5}$ found a specificity of $8.92 \%$. Research by Wong et al. ${ }^{15}$ found a specificity for barium enema of $82 \%$. The significant differences in the results of the sensitivity and specificity tests in this study could be influenced by several factors, including a larger sample size compared to similar studies in the infant age range $(\leq 12$ months). Second, the variation in age characteristics was narrower and the mean age was younger compared to other studies that tested the accuracy of barium enema as an early diagnostic tool for Hirschsprung's disease. In the study of Huang et al. ${ }^{5}$ used a sample of 22 people with an average age of 2.4 years, while Wong et al. ${ }^{15}$, conducted a similar study with a sample size of 43 people and a mean sample age of 43 days. Research by Abbas et al. ${ }^{11}$ was even carried out on 60 samples of patients with a much older mean age of $3.5+0.5$ years. Another factor that influenced the results of this study was the barium enema examination procedure was not performed by one radiologist for all patients, but was performed by several radiologists so that there may be differences in interpretation of the barium enema photo reading.

The level of accuracy in this study was $94.2 \%$. This shows that the barium enema examination has the ability of $94.2 \%$ to determine whether the subject has 
Hirschsprung's disease or not. The level of accuracy in this study is much higher than that of Abbas et al., ${ }^{11}$ which is $76.67 \%$. Based on this level of accuracy, it can be believed that a barium enema radiological examination can be used as an early diagnostic tool in Hirschsprung's disease in the infant age group.

The positive predictive value in this study was obtained at $97.7 \%$. This indicate that from 100 people, barium enema could detect Hirschsprung's disease in 98 people. Meanwhile, the negative predictive value obtained was $77.8 \%$, indicating that in a population of 100 people the ability of the barium enema examination to detect no Hirschsprung's disease was 78 people. This result is much higher when compared to the results of research conducted by Wong et al. ${ }^{15}$ which obtained a positive predictive value of $41 \%$ while a negative predictive value of $100 \%$.

Based on the results of the above studies, barium enema examination can be used as one of the initial examinations for infants who are suspected of having Hirschsprung's disease before histopathological examination, either through rectal biopsy or post-definitive surgery. Researchers also assessed the advantages possessed by the barium enema examination, namely that it is easier to perform and cheaper and the most important thing is that this examination is not invasive to be used as an early diagnostic tool in the infant age. If the barium enema examination is positive, it is followed by histopathological examination as the gold standard.

A weakness of the study relates to the low incidence of disease cases. This led to a retrospective research, which is hospital-based research. Researchers took a longer time span because of the very limited number of infant patients who developed Hirschsprung's disease in the span of one year. In addition, the incomplete medical record recording system also made it difficult for researchers, that many samples were excluded and extended the sampling time span.

\section{CONCLUSION}

Barium enema has high sensitivity, specificity, and accuracy in diagnosing Hirschsprung's disease. Barium enema has good diagnostic value in diagnosing Hirschsprung's disease.

\section{ACKNOWLEDGEMENT}

Author thanked all research participants.

\section{DISCLOSURE}

No conflict of interest exists regarding to this research.

\section{REFERENCES}

1. Gunnarsdottir A, Wester T. Modern Treatment of Hirschsprung's Disease. Scand J Surg. 2011;100:243-9.

2. Yan Z, Poroyko V, Gu S, et al. Characterization of the intestinal microbiome of Hirschsprung's disease with and without enterocolitis. Biochem Biophys Res Commun. 2014;445:269-74.

3. Esayias W, Hawaz Y, Dejene B, et al. Barium Enema with reference to rectal biopsy for the diagnosis and exclusion of Hirschsprung's disease. East Cent Afr $J$ surg. 2013;18:143-7.

4. Georgeson KE. Chapter 35: Hirschsprung's Disease. In: Holcomb G, Murphy JP, editors. Ashcraft's Pediatric Surgery. $5^{\text {th }}$ Edition. Philadelphia: Elsevier; 2010. p.456-67.

5. Huang CC, Shih SL, Chen YF, et al. Hirschsprung Disease and Contrast Enema: Diagnostic Value of Simplified Contrast Enema and Twenty-Four-HourDelayed Abdominal Radiographs. $J$ Radiol Sci. 2011;36:159-64. 
6. Kessmann J. Hirschsprung's disease: Diagnosis and Management. Am Fam Physician. 2006;74:1319-22.

7. Amiel J, Lyonnet S. Hirschsprung disease associated syndromes, and genetics: a review. J Med Genet. 2001;38:729-39.

8. Moore SW, Tsifularo N, Nmadu PT, et al. Chapter 76: Hirschsprung's Disease. In: Ameh EA, Bickler SW, Lakhoo K, et al, editors. Pediatric Surgery: A Comprehensive Text for Africa. Seattle: Global HELP; 2011. p.448-54.

9. Nurko S. Hirschsprung's Disease. Motility Society [serial online] 2006 [cited 2015 November 29]. Available from: http://www.motilitysociety.org/pdf/Hirsc hsprung's\%20 disease\%208.28a.2006.pdf 10. de Lorijn F, Kremer LCM, Reitsma JB, et al. Diagnostic Test in Hirschsprung's Disease: A Systematic Review. J Pediatr Gastroenterol Nutr. 2006;42:496-505.

11. Abbas M, Rashid A, Laharwal AR, et al. Barium Enema in the Diagnosis of
Hirschsprung's Disease: A Comparison with Rectal Biopsy. Arch Clin Exp Surg. 2013;2:224-8.

12. Saravanan K, Kumaran V, Rajamani G, et al. Minimally invasive pediatric surgery: Our experience. J Indian Assoc Pediatr Surg. 2008;13:101-3.

13. O'Donovan AN, Habra G, Somers S, et al. Diagnosis of Hirschsprung's Disease. AJR Am J Roentgenol. 1996;167:517-20.

14. Ishfaq M, Ahmad UF, Manzoor S. Hirschsprung Disease; Diagnosis and Management: Experience at Ibn-E-Siena and Nishtar Hospital, Multan. Professional Med J. 2014;21:20-6.

15. Wong AWY, Tsang DSF, Lam WWM. How Useful is Contrast Enema in the Diagnosis of Hirschsprung's Disease? Five-year Experience from a Local Referral Centre. Hong Kong J Radiol. 2014;17:30-5. 